\title{
Dielectric properties of an eco-friendly ferroelectric nanocomposite from cellulose nanoparticles mixed with Rochelle salt
}

\author{
Bich Dung Mai ${ }^{1}$, Hoai Thuong Nguyen ${ }^{2,3, *}$, A.Yu. Milinskiy ${ }^{4}$, S.V. Baryshnikov ${ }^{4}$ \\ ${ }^{1}$ Institute of Biotechnology and Food Technology, Industrial University of Ho Chi Minh City, \\ 700000, Ho Chi Minh City, Vietnam \\ ${ }^{2}$ Division of Computational Physics, Institute for Computational Science, Ton Duc Thang University, \\ Ho Chi Minh City, Vietnam \\ ${ }^{3}$ Faculty of Electrical \& Electronics Engineering, Ton Duc Thang University, Ho Chi Minh City, Vietnam \\ nguyenhoaithuong@tdtu.edu.vn \\ ${ }^{4}$ Faculty of Physics and Mathematics, Blagoveschensk State Pedagogical University, \\ 675000, Blagoveschensk, Russia
}

The idea of this study is to develop an eco-friendly ferroelectric nanocomposite based on cellulose nanoparticles (CNP) prepared from cotton waste (Fig. 1a). In [1, 2] the the wood was utilized to improve the piezoelectric response of Rochelle salt (RS) or matrix of porous cellulose to synthesize composite with RS. However, due to difficulties in controlling the filling level because of using porous matrices with nanochannels, the influence of RS content on composite properties was not discussed. Besides, ferroelectric properties of RS as phase transition and dielectric relaxation at low frequencies were not reported. To solve these problems, a mixed composite at different composition weight ratios was synthesized in the present study. The composite was carefully characterized to explore the changes of structure and functional groups, and therefore to clarify the interaction of components in the composite (Fig. 1b).

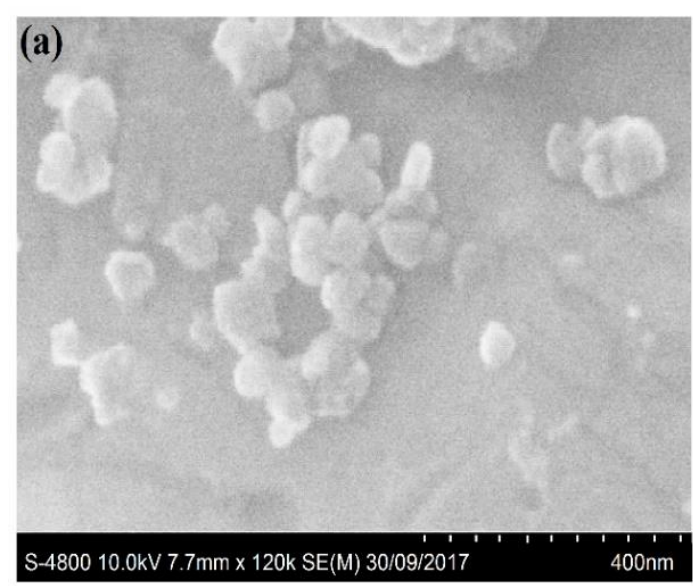

(a)

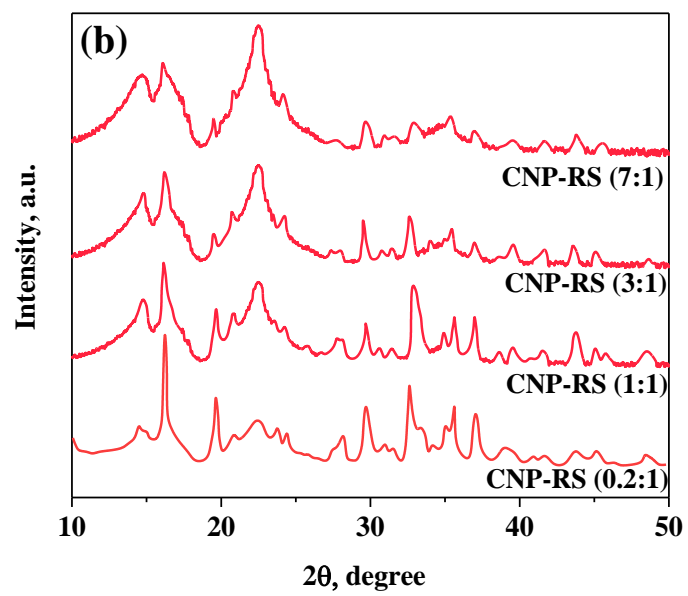

(b)

Figure 1. (a) SEM image of the used cellulose grown in near spherical shape and (b) XRD spectra of CNP-RS composite at different composition weight ratios.

The results showed that at low CNP content $(\mathrm{CNP}: \mathrm{RS}=0.2: 1)$ the upper Curie point $\left(24^{\circ} \mathrm{C}\right)$ characteristic for the RS cluster was observed. For CNP:RS of 0.5:1, 1:1, 3:1 and 5:1, two phase transitions points: the lower-temperature one related to RS and the higher-temperature one - to CNP shifted to higher temperatures with increasing CNP content due to the stronger interaction between CNP and RS components were detected. Especially, at CNP:RS ratio of 7:1 and higher CNP content, there was no phase transition detected owing to the maintenance of ferroelectric phase up to decomposition temperature. Besides, a reduction of relaxation frequencies with increasing CNP content was also observed. The strong interaction between hydrogen containing $\mathrm{CNP}$ and RS was assumed to be a reason for these mentioned anomalies.

1. E. Lemaire, C. Ayela, A. Atli. Smart Materials and Structures 27, 2 (2018).

2. A.S. Sidorkin, S.D. Milovidova, O.V. Rogazinskaya, Hoai Thuong Nguyen. Patent RU2666857C1 (2018). 